\title{
Analysis of the value chain of sheep wool in spain and its possible use in greenhouse crops
}

\author{
Laura Marcos Bilbao', Julián Briz Escribano², Stefan Köhler ${ }^{3}$ \\ ${ }^{1,2}$ Universidad Politécnica de Madrid (UPM) - Avda. Complutense, s/n. 28040, Madrid - Espanha. \\ ${ }^{3}$ Institut für Agrar- und Stadtökologische Projekte (IASP) - Philippstrasse 13 Haus 16, D-10115 Berlin. \\ Email: lauramarcosb@gmail.com, julian.briz@upm.es, stefan.d.koehler@agrar.hu-berlin.de
}

Received: February $25^{\text {th }}, 2018$.

Accepted: March $10^{\text {th }}, 2018$.

Published: March $30^{\text {th }}, 2018$.

Copyright (C2016 by authors and Institute of Technology Galileo of Amazon (ITEGAM).

This work is licensed under the Creative Commons Attribution International

License (CC BY 4.0).

http://creativecommons.org/licenses/by/4.0/ (c) (i) (2) Opea Aces:

\begin{abstract}
The importance of wool production has declined in recent decades due to competition from other textile fibers. Despite its many positive attributes, wool has been in the background of textile fibers, in part due to a lack of consumer information, lack of interest from industry, abusive margins on final prices, lack of transparency in the sector... The lower value of the sheep wool in Spain has originated the closing of many traditional industries, the decrease in livestock, lack of interest in wool production by farmers, considering it as a waste... Considering that wool production is a must in sheep farm, measures are needed to obtain the maximum economic benefit. The study of the value chain of sheep wool is needed in order to understand the sector structure, its behavior and its performance, to be able to offer potential uses for wool with no interest to the textile industry.
\end{abstract}

Key Words: Value chain, Sheep wool, Greenhouses, Woolvation blocks.

\section{Análisis de la cadena de valor de la lana de Ovej a en España y su posible uso en cultivos de invernadero}

\begin{abstract}
RESUMEN
La importancia de la producción de lana ha disminuido en las últimas décadas por la competencia de otras fibras, tanto sintéticas como de origen animal o vegetal. A pesar de los muchos atributos positivos que tiene, la lana ha quedado en un segundo plano dentro de las fibras textiles, en parte debido a la falta de información de los consumidores, desinterés por parte de la industria, márgenes abusivos en los precios finales, falta de transparencia del sector... En España la reducción del valor de la lana ha provocado el cierre de muchas empresas transformadoras tradicionales, disminución de las cabezas de ganado, desinterés de la producción de lana en las explotaciones llegándola a considerar un subproducto, teniendo en cuenta que es una producción obligada en la explotación ovina, es necesario adoptar medidas para obtener el máximo beneficio económico. El estudio de la cadena de valor de la lana de oveja es necesario para conocer la estructura del sector, su conducta y el funcionamiento para así poder proponer posibles usos a la lana que no es de interés para la industria textil. del prototipo sobre PROTEUS, emulando también la electrónica. Además, se grafican los esquemas electrónicos simulados del sistema, se brindan datos de parámetros y el estado de las alarmas.
\end{abstract}

Palabras Claves: Cadena de valor, Lana de oveja, Invernaderos, Woolvation blocks.

\section{INTRODUCCIÓN}

La lana es una fibra natural con un linaje verdaderamente verde, siendo tanto sostenible como biodegradable, además de gozar de otras ventajas que le han hecho ganar una reputación de alta calidad. Su compleja estructura le asegura su habilidad natural para respirar, en forma única absorbe y elimina humedad. $\mathrm{Su}$ elevado contenido en agua y nitrógeno hacen de la lana un retardante del fuego sin necesidad de tratamientos químicos, por lo que se puede considerar como una fibra segura, además absorbe emisiones insanas de carbono en la atmósfera. Se la encuentra en muchos sectores: vestimenta y moda, ropa deportiva, alfombras y usos interiores, aviación, arquitectura, sector manufacturero, uso médico y prendas de protección. Por su versatilidad dinámica se considera la lana como una fibra inteligente. A pesar de todos estos atributos positivos, los 
consumidores, la industria y los gobiernos generalmente se olvidan de los beneficios que la lana general en materia de salud yseguridad [1]. En los últimos años la lana ha pasado de ser un producto significativo a uno secundario, el precio pagado es el principal problema, y esto ha hecho que los ganaderos pierdan el interés por conseguir mejoras en la producción.

El objetivo principal de la producción ovina en España, y en Europa en general, es la leche o a la carne, y la lana se considera un coste asociado a la producción de la explotación. Teniendo en cuenta que la lana es una producción obligada en la explotación ovina es muy importante tomar medidas para hacer de este subproducto lo más económico posible [2].

Actualmente el ganadero no puede pagar el esquileo de los animales con el precio de venta de la lana, y el esquileo debe realizarse por cuestiones higiénicas. Según los datos obtenidos en las encuestas realizadas a diferentes ganaderos, el precio medio de esquila por oveja es de entre 1 y $4 €$. El precio aproximado que se paga por el $\mathrm{kg}$ de lana es de $0,60 €$.

Ante el poco estímulo económico que supone la lana el ganadero se esfuerza poco en la obtención, clasificación y comercialización de la misma. Además el papel del ovino, que suele estar en zonas agroclimáticas menos favorables, es esencial para mantener la actividad agraria y que el manejo tradicional de rebaños de ovino y caprino asegura la presencia humana en las zonas rurales menos favorecidas, evitando así la despoblación y contribuyendo a mantener en buenas condiciones dehesas, montes, prados y rastrojeras [3].

Teniendo en cuenta todo esto, la industria lanera necesita apoyo para remediar esta amenaza a su existencia. Por lo comentado anteriormente, desde numerosos organismos como el Ministerio de Agricultura, Alimentación y Medioambiente (MAGRAMA) español, la Asociación Agraria de Jóvenes Agricultores (ASAJA) o el Instituto de Proyectos de Ecología Agraria y Urbana (IASP) afiliado a la Universidad Humboldt de Berlín se empezaron a plantear desde hace un tiempo cómo cambiar la situación actual de la lana de oveja.

Se han realizado algunos estudios y el presente trabajo surgió como fruto de la colaboración entre la Universidad Politécnica de Madrid (UPM) y la Universidad Humboldt de Berlín, creyendo que analizar la Cadena de Valor de la lana de oveja en España podría ayudar a ver qué eslabones son críticos o tienen un mal funcionamiento y proponer soluciones.

Gracias a la investigación realizada en el IASP y los experimentos llevados a cabo en los campos de prácticas de la UPM, se propone una posible solución a la utilización de la lana de oveja que queda como subproducto y aprovecharlo en el sector de los cultivos forzados.

\section{OBJETIVOS}

Los objetivos principales del estudio son: mejorar la eficiencia de la cadena de valor de la lana de oveja analizando los diferentes eslabones e incrementar el poder negociador de los ganaderos. Los derivados o secundarios son la reutilización de la lana sin salida al mercado, abastecer a invernaderos y cultivos forzados de material para sustrato y fomentar una cadena de valor sostenible.

\section{MATERIALES Y MÉTODOS}

Metodología GLOCAL de análisis de la cadena de valor.

La cadena de valor, concepto introducido por Michael Porter en su libro "Competitive Advantage: Creating and Sustaining Superior Performance (1985)”, es un modelo teórico que permite describir el desarrollo de las actividades de una organización empresarial generando valor al cliente final y a la misma empresa [4]. Cada empresa es un conjunto de actividades que se realizan para diseñar, producir, comercializar y apoyar a sus productos [5].

Todas estas actividades pueden ser representadas mediante la cadena de valor, y se clasifican en primarias (producción, logística, marketing de ventas y servicio postventa) y de apoyo (infraestructura empresarial, recursos humanos, desarrollo tecnológico y aprovisionamiento) debiendo estar todas ellas coordinadas.

La metodología GLOCAL trata de combinar una visión global de la cadena de valor con una actuación específica local. La estructura del estudio a nivel global incluye tres grupos de factores innatos en la organización y desarrollo de la cadena de valor: la estructura, la conducta y el funcionamiento. La estructura se refiere al sistema organizativo, tanto empresarial como institucional, en la cadena de valor, disponiendo de una serie de dimensiones que permiten evaluar y contrastar situaciones diferentes. Se estudian: la organización empresarial en cada uno de los eslabones de la cadena, relaciones entre los actores de la cadena y su forma organizativa y la diferenciación del producto a lo largo de la cadena de valor.

La conducta recoge el comportamiento de los agentes sociales y económicos que operan en la cadena de valor y se relacionan con cuestiones éticas y morales. El funcionamiento refleja el resultado de cómo desempeñan sus funciones los distintos elementos de la cadena de valor. Se considera el resultado que aporta la cadena como consecuencia de la estructura y conducta existentes.

Identificando criterios que permitan evaluar y comparar diversas capacidad de adaptación. Por último el enfoque local trata de responder a una cuestión o preocupación específica realizando estudio de tendencia, ciclos o estacionalidad, evolución de tipos de empresas, entrevistas a los agentes de los diferentes eslabones, análisis comparativos nacionales o internacionales o estudio de la interacción entre actores de la cadena o entre cadenas [6].

\section{III.1 MATERIALES}

Como fuentes primarias de información destacan las encuestas realizadas a los distintos eslabones de la cadena de la lana de oveja. La labor de ponerse en contacto con productores, transformadores o consumidores no fue sencilla debido a la escasa transparencia en el sector, aún así la información recogida aportó información relevante. Se realizaron encuestas a ganaderos, para obtener información del eslabón productivo.

De entre todas las Comunidades Autónomas de España, se descartó Extremadura ya que su producción principal es oveja merina y su lana tiene alta calidad, se comercializa bien y por ello no se considera un subproducto. Había muchas opciones, pero por posibilidades de contactos se eligió Castilla y León, que además es la segunda Comunidad Autónoma con mayor censo ovino de España, con casi un $20 \%$ del total nacional.

Se realizaron 27 encuestas en diferentes provincias. También se realizaron 2 encuestas en la Comunidad de Madrid. Además se visitó uno de los pocos lavaderos de lana que quedan en España, en Mota Del Cuervo, entrevistando al propietario. También se visitaron 2 fábricas de hilo de lana, una en Sonseca (Toledo) y otra en Mota Del Cuervo, pudiendo hablar con los propietarios y con trabajadores.

En las visitas se pudo comprobar el proceso de transformación que sufre la lana desde la esquila en campo y la 
recogida de la lana (por personal del propio lavadero), el sorteo o clasificación de la lana, lavado, secado, hilado, etc. La información recogida fue muy útil, ya que de primera mano se pudo saber el rendimiento de cada operación y la cantidad de desperdicios que se producen.

La propuesta de este trabajo para un futuro posible para la lana de oveja, es su utilización como sustrato para cultivos forzados. Por ello, se creyó oportuno realizar unas encuestas a algunos invernaderos en Murcia, Almería, Madrid y Burgos. Y así poder comprobar qué sustrato estaban usando, qué aspectos del sustrato les parecen más relevantes, si estarían dispuestos a probar nuevos sustratos como la lana, etc.

Las fuentes secundarias de información utilizadas para redactar este documento, han sido principalmente trabajos, proyectos, estudios y documentos promovidos o elaborados por el Ministerio de Agricultura, Alimentación y Medio Ambiente (MAGRAMA) español. Algunos de ellos se han podido obtener de la propia página web del Ministerio. Otros han sido proporcionados por colaboradores que trabajan en la Asociación Agraria de Jóvenes Agricultores (ASAJA) de Madrid y Burgos. La mayoría de los datos de producción, censos, consumo y exportaciones e importaciones se han obtenido de la web de FAOSTAT, donde se localizan las estadísticas de la FAO (Food and Agriculture Organization of the United Nations).

\section{ANÁLISIS DE LA CADENA DE VALOR DE LA LANA DE OVEJA}

\section{IV.1 ESTRUCTURA DEL MERCADO DE LA LANA}

Como norma general, la cadena de comercialización de la lana se puede dividir en tres eslabones fundamentales: productores, transformadores y consumidores.

El primer eslabón de la cadena es la producción, localizada en las explotaciones ganaderas, es el productor o ganadero el que suele vender sus partidas de lanas a tratantes comarcales que se encargan de su transporte y almacenamiento, pudiéndola vender también directamente a mayoristas. Se analizan a continuación los censos y la producción de lana a nivel mundial, europeo y español.

En el 2012, según los datos de FAOSTAT, el censo de ovino mundial estaba entorno a 1.170 millones de cabezas de ganado. Según estos datos China con 187 millones de cabezas, se sitúa a la cabeza (16\% del total de cabezas), seguido de la India con 75 millones aproximadamente $(6,42 \%$ del total). España ocupa el puesto diecinueve con un total de 16,8 millones de cabezas (1,44\% del total). El censo de ovino en Europa en 2012 se situó alrededor de los 129 millones de cabezas de ganado. A partir de 1990 se produce un decrecimiento pronunciado en el censo hasta 2001, con una pérdida de unos 125 millones de cabezas. En los últimos 13 años se mantuvo más o menos constante [7].

A nivel europeo, destacan principalmente Reino Unido, Rusia, España y Grecia que ocupan las primeras posiciones. La importancia relativa de sus cabañas sufrió un reajuste en 2007, tras la incorporación de Rumania y Bulgaria. Rumania irrumpe con fuerza en el escenario de los pequeños rumiantes y pasa a ser la quinta cabaña en importancia, por delante de las de Francia e Italia. Reino Unido con 32,2 millones de cabezas de ovino y participa en la Unión Europea de los 27 con un porcentaje del $33 \%$. España ocupa el segundo lugar con una participación del $17 \%$ y un censo próximo a los 16,8 millones de cabezas. La incorporación de Rumania y Bulgaria (10\% del censo) corrigió ligeramente la evolución a la baja de la cabaña comunitaria en su conjunto [8].

Según el Sistema Integral de Trazabilidad Integral español (SITRAN) el censo total de ganado ovino a 1 de diciembre de 2013 era de 16.609.069 cabezas en España [9].

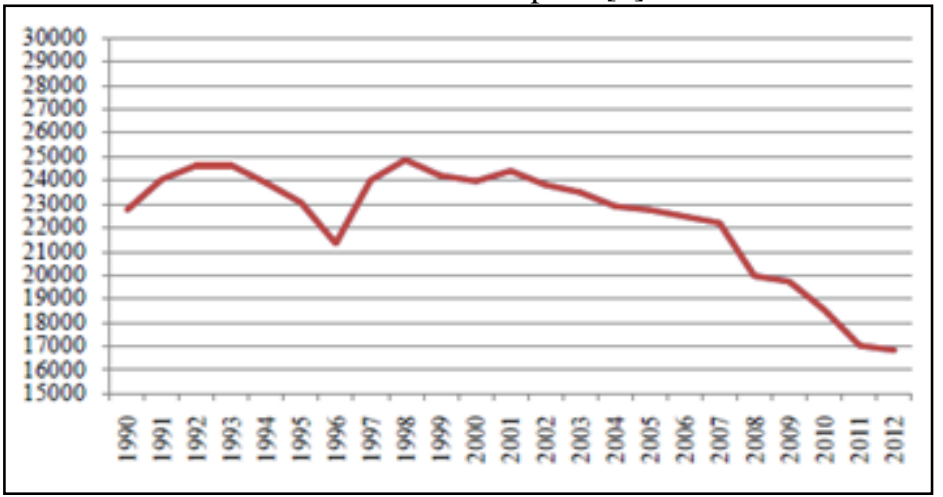

Figura 1: Evolución del censo de ovino en España, en miles de cabezas (1990-2012).

Fuente: [10].

La producción de lana lleva disminuyendo ya bastantes años por la competencia de otras fibras naturales vegetales, como el algodón y el lino, y sobre todo por las fibras sintéticas derivadas de la industria del plástico (acrílicas, etc.).

La producción mundial de lana se sitúa en torno a las 1.200 millones de toneladas, con una ligera variación anual y una tendencia a la baja entre el año 2002 y 2007 , del orden de 5,5\%. En relación a la producción mundial de fibras, la lana limpia representa el $1,73 \%$ de la producción mundial y ocupa el tercer lugar, muy por debajo de las fibras sintéticas $(59,3 \%)$ y del algodón $(37,3 \%)$ [11].

La producción de lana en sucio en el mundo asciende a 2.066.695 toneladas, en el año 2012 según datos de FAOSTAT [12]. Asia y Oceanía aportan el $68 \%$ de la producción de lana sucia del mundo, es decir más de la mitad de la lana sucia del mundo se produce sólo en dos continentes y Europa produce un $13 \%$.

Cabe destacar la tremenda disminución de producción de lana sucia mundial si comparamos la producción en el año 1990 que era de 3.350.508 toneladas, con la producción en el pasado año 2012 que fue de 2.066.695 toneladas. Estamos hablando de una pérdida de 1.283.813 toneladas de lana sucia producida en 22 años [13]. En cuanto a la producción mundial de lana en sucio por países, China, Australia y Nueva Zelanda se sitúan a la cabeza, y entre las tres capitalizan casi la mitad del total mundial, un $45 \%$. España ocupa la posición número 20 con casi 23 toneladas de lana sucia en el año 2012, lo que supone una aportación del 1,11\% a la aportación mundial de lana en sucio [14].

La producción en Europa de lana en sucio en 2012, asciende a 267.131 toneladas, lo que supone una aportación a la producción mundial del 13\% como se ha comentado. En cuanto a la aportación por países, el mayor productor es el Reino Unido con un $25 \%$ de la producción europea, en segundo lugar se encuentra Rusia con un $20 \%$ y en tercer lugar España, que aporta un $8 \%$ [15]. 
Bilbao, Escribano and Köhler, ITEGAM-JETIA. Vol. 04, № 13, pp 109-116. March, 2018.

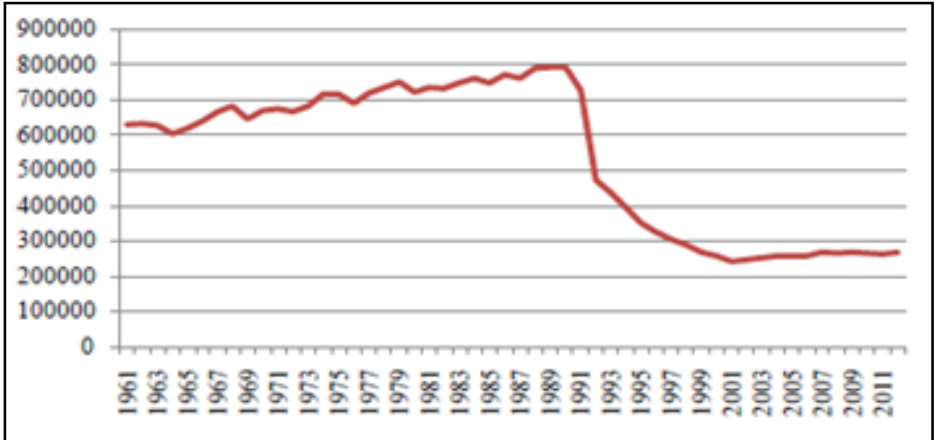

Figura 2: Evolución de la producción de lana en Europa, en toneladas (1990-2012).

Fuente: [16].

España se mantiene como tercer productor europeo, tanto en lana sucia como en limpia, por detrás del Reino Unido y Rusia, seguido por Rumania y Francia, como ya se ha indicado. En números absolutos, ambas producciones acusaron un descenso importante a partir del año 2000.

La producción de lana bruta en España en 2012 alcanza unas 22.584 toneladas, de las que 4.341 son de lanas finas, 12.963 de entrefinas y 5.102 de bastas, lo que representa un $19 \%$, un $57 \%$ y un $23 \%$ respectivamente de la producción española. Las lanas negras representan solo unas 178 toneladas, un $1 \%$ de la producción total [17].

Los datos de producción de lanas españolas deben interpretarse según criterios de su aplicación textil. En efecto, no todas las lanas tienen un interés para la industria y ello repercute en consecuencia en su apreciación económica. Uno de los retos es encontrar alternativas para el uso de tales lanas "no textiles" y evitar el considerarlas como subproducto (vertederos, incineración,...). Debemos adaptar nuestras lanas a las necesidades de los clientes y trabajar con materiales y procesos más sostenibles [18].

El eslabón de manufactura incluye el proceso de obtención de lana en las explotaciones y el proceso industrial que debe sufrir para ser apto para la industria. El ciclo productivo de la materia prima es anual y está caracterizado por tres etapas: el marcado, la cubrición y la esquila. Dentro de estas etapas se desarrollan diversas tareas, que implican distintos grados de especialización.

El marcado y la cubrición están consideradas dentro de la actividad ganadera y la duración de cada una de ellas está determinada por el proceso biológico del animal y por la especialización de los productores en cada una de ellas, como por ejemplo si realizan o no inseminación artificial.

Las tareas realizadas pueden ser llevadas a cabo por el propio ganadero o sus empleados, o contratar a terceros para tareas específicas. El esquileo, el acondicionamiento y el envasado se deben realizar en un lugar de esquila adecuado para tales fines. Para revalorizar la calidad de la lana y mejorar su competitividad en el mercado hay que tener como prioridad la satisfacción de los requisitos deseados por los clientes y beneficiarios.

Para ello es necesaria la adopción masiva de algunas prácticas tecnológicas como por ejemplo la esquila Tally-Hi, de alto rendimiento, permite una esquila correcta, de alta calidad y con menor esfuerzo que cualquier otro método. Su método de enseñanza prioriza la calidad frente a la velocidad, por lo que es fácil de aprender. Si se esquila de forma correcta por este método, todas las partes se benefician tanto el ganadero, la cuadrilla y el esquilador como la industria textil.

Otras prácticas tecnológicas son: el acondicionamiento básico de la lana en el almacén, el envasado en material no contaminante y el análisis en laboratorio de la calidad de las lanas producidas. Es muy importante hacer hincapié en la obtención de vellones limpios y libres de contaminantes, como lana pigmentada (negra, de lunares), coloreada (manchada de orina, con pinturas no lavables), con problemas de coloración (lanas amarillas).

Con el acondicionamiento también se evita la incorporación de toda clase de materiales extraños, plásticos, hilos, colillas y alambres, además se separan los vellones en un mínimo de clases de lana dentro del lote. El envasado se realiza en fardos o sacas de polietileno de 200 micrones o de cualquier otro material similar.

El análisis de laboratorio es una buena herramienta para mejorar la producción, es muy importante que los productores conozcan con exactitud las principales características de su lote ya que influyen directamente sobre el precio que van a percibir por él. Las ventajas de realizar la medición de dichas características sobre una muestra representativa del lote son: permitir al ganadero conocer las virtudes y defectos del lote, pudiendo así decidir los cambios de manejo que permiten mejorar su producción, se puede conocer con mayor certeza y exactitud el valor de la lana, el uso de la información objetiva es una condición necesaria a la hora de comercializar un lote de lana.

Utilizando el análisis de ensayo tradicional, también denominado método de muestra de calado o Core Test, se miden las siguientes características: diámetro medio de fibras o finura que es el promedio de los diámetros de las fibras limpias de lana y se determina por el método "Air Flow" (es la característica más importante desde el punto de vista comercial y determina el precio alcanzado). También, el porcentaje de materia vegetal adherida a la lana que no debe superar $1,5 \%-1,8 \%$ en una buena lana, ya que al incrementarse disminuye el rinde al peinado que es el valor más importante comercialmente hablando en un lote de lana sucia y se relaciona con su rendimiento industrial en el peinado. Por último se mide el rinde al lavado es el rendimiento de la lana limpia sobre lana sucia lavada. Una vez esquilada, la lana es adquirida por los compradores que llevan a cabo su industrialización en diferentes grados.

El proceso completo, desde la recepción de la lana sucia hasta la obtención de la lana hilada lista para fabricación de tejidos, pasa por las siguientes etapas: Clasificación: es la separación de los vellones por finura, determinación del tipo industrial y eliminación de partes de los vellones que no son aptos para la posterior transformación. Se realiza en las plantas manufactureras sobre las lanas sucias o grasientas. Prensado: en esta etapa se prensa la lana (tanto sucia, lavada o peinada) con una máquina hidráulica para disminuir su volumen y facilitar su transporte, en forma de fardos. Lavado: sirve para separar de las fibras la grasa y otras sustancias, pero sin remover la materia vegetal.

Primero se trata la lana para la licuación de contaminantes grasientos, después se añade un material capaz de absorber estos contaminantes licuados. Secado: se realiza mediante corrientes de aire caliente, en una máquina secadora centrífuga. Eliminación de las materias vegetales: se debe hacer sin afectar a las fibras, se realiza a través de medios químicos o mecánicos. Cardado: se usa una máquina que transforma las fibras en mechas circulares que después se arrollan en bobinas.

Posteriormente se hace pasar la lana por las cardas, sección que está compuesta por máquinas de tambores grandes y pequeños de distintas velocidades, con púas de acero de medidas adecuadas para separar las fibras y eliminar la mayor parte de los vegetales o semillas.

El producto sale en grandes lotes de mecha cardada. Intersecting: hacen una homogeneización de las mechas para la 
entrada a las peinadoras. Peinado: se elimina la fibra corta de lana o restos vegetales, y se paralelizan las fibras largas hasta formar la mecha de peinado, llamado tops, que puede pasar a la hilandería tal cual, o previo teñido, o comercializarse hacia otras industrias.

Preparación: con máquinas intersecting y bobinadoras, acondicionan la mecha haciéndola más homogénea para su posterior hilatura, se afinarán hasta el título o grosor adecuado para la clase de hilado a elaborar. Hilado: es la etapa previa para el uso industrial y consiste en el estirado, la torsión y el plegado. En lanas cardadas el estirado llega a cuadruplicar su longitud original, mientras que en lanas peinadas puede llegar de seis hasta dieciséis veces. Con la torsión necesaria aplicada sobre la lana estirada se termina el hilo en un cabo con las "maquinarias continuas de hilar", que puede seguir el proceso de fabricación o comercializarse así. Retorcido: se utilizan máquinas continuas que dan la torsión requerida, para aumentar tanto el grosor como la resistencia del producto.

Otra vez, puede seguir el proceso de fabricación o comercializarse. Madeja: con un aspa se recoge la lana hilada en vueltas iguales, se lava otra vez con jabón para eliminar residuos. Tras este proceso se puede usar lana en su color natural o se puede teñir. Teñido: se selecciona el producto del que se desea obtener el color, se hierve en agua y se agrega a la lana mojada. Tras un periodo de cocción se añade una sustancia que fije el color (sal, vinagre, piedra lumbre o sulfato de cobre). Tejido: tras sufrir todos los procesos anteriormente explicados la lana será apta para realizar los productos que se encuentran en el mercado [19].

Desde siempre la industria textil ha sido el principal demandante de lana, pero ésta ha ido disminuyendo su participación en el mercado de la indumentaria con el tiempo, quedando relegada por otras fibras de origen vegetal como el algodón y de origen sintético como las microfibras que respondieron rápidamente a los requisitos del consumidor final.

Para satisfacer de una forma adecuada a los usuarios se produjeron cambios tecnológicos desde el sector productivo lanero en mejora genética, manejo del ganado y de los recursos alimentarios, esquila, etcétera produciendo un alto impacto en determinadas propiedades de la lana, que medidas objetivamente demuestran una clara mejora cualitativa y de precio del producto.

Es necesario que el sector productivo y los procesadores industriales dirijan todos sus esfuerzos a satisfacer los requisitos del usuario. Éste requiere una prenda de vestir que sea liviana y cómoda, de buen diseño, informal, suave al tacto, confortable para usar en contacto con la piel y que tenga fácil cuidado y mantenimiento.

En 2007 el consumo final de lana virgen en el mundo fue de 1.231 millones de kilogramos y según un informe elaborado por la IWTO (International Wool Textile Organisation) el principal uso de la lana $(66 \%)$ es en la fabricación de prendas (vestimenta), seguido por el uso en interiores (30\%), que incluye alfombras, tapetes y tapicería.

Por último, el uso industrial de la lana es del 4\%. La lana es una fibra multifuncional en un amplio rango de diámetros, algunos ejemplos pueden ser: Vestimenta: trajes, sacos sport, pullovers, ropa deportiva, ropa de abrigo para la nieve, bufandas, guantes, medias, sombreros, ropa interior, ropa para niños, ropa interior térmica, telas resistentes al agua, prendas informales, zapatos, ropa formal, hilados para tejer a mano, uniformes...

Usos interiores: alfombras, edredones, colchones, frazadas, tapicería, tapices, lámparas, sillas, tapetes, acolchados, cubrecamas, manteles, cobertores de pared, sofás, filtros... Textiles técnicos: interiores de aviones, aislante térmico, aislante de sonido, aislantes de techos, filtros, prendas resistentes al fuego, uniformes de policía, uniformes militares, aire acondicionado, prevención de lesiones secundarias en la piel, filtros para pianos, filtros para controlar polvo y olores químicos, telas de billar, componentes de automóviles, pelotas de tenis...[20].

\section{IV.1.1 CONDUCTA DE LOS PRINCIPALES AGENTES DE LA CADENA}

Hay una evidente falta de transparencia en la información de mercado en la cadena de valor de la lana de oveja en España.

Por ello la conducta de los agentes que se presenta a continuación es una interpretación de los datos encontrados y de los comentarios obtenidos mediante las visitas y encuestas realizadas.

Los ganaderos perciben poco dinero por la venta de la lana y los lavaderos y las cardadoras perciben algo más por el valor añadido proporcionado al producto, teniendo en cuenta los precios tan elevados que alcanza el producto final se puede considerar que debe de haber márgenes abusivos en la etapa final del producto. Esto afecta negativamente a los consumidores que dejan de comprar los productos elaborados a partir de lana, y en consecuencia a los ganaderos que reciben cada vez menos dinero por ella.

Esto implica que en las explotaciones se dediquen cada vez menos recursos a mejorar la calidad de la lana de sus ovejas. Por ejemplo, se descuida el proceso de selección de la lana en campo mezclándose lanas y haciendo más difícil la tarea de sorteo en la lavadora.

También se descuida la limpieza de las instalaciones que afectará al contenido de contaminantes o partículas no deseadas que contenga la lana como manchas de orín, restos de excrementos, materias vegetales,... No se pondrá atención en los productos utilizados en el marcado de los animales, ni se realizará la esquila adecuada por no poder invertir dinero en algo que no va a ser remunerado.

Todo esto influye en el estado en el que llega la lana a las siguientes etapas de manufactura provocando el exceso de material que hay que desechar por no ser válido para entrar en las lavadoras. Se considera fundamental la participación de las Administraciones públicas en la regulación de las conductas de producción de lana en las explotaciones y en recopilar información veraz de cada eslabón y ponerlas a disposición de los interesados.

Esto mejoraría la calidad de la lana en las explotaciones ganaderas y también la transparencia del sector proporcionando información útil en todos los eslabones, incluido el consumidor que debería ser consciente del producto que desea adquirir, el proceso de elaboración que ha sufrido, la materia prima de la que procede y los atributos positivos de los que goza. Además los productores conocerían los deseos y preferencias de los consumidores y les daría la oportunidad de adaptar el producto.

\section{IV.1.2 FUNCIONAMIENTO DE LA CADENA DE VALOR}

El funcionamiento, ya definido anteriormente, refleja el resultado de cómo desempeñan sus funciones los distintos elementos de la cadena de valor.

Es el resultado que aporta la cadena como consecuencia de la estructura y conducta existentes. Un criterio que puede ayudar para entender el funcionamiento en la cadena es la eficiencia económica, que se puede definir como la relación beneficio-coste de un producto o un servicio generado.

En el caso de la cadena de valor de la lana de oveja, la eficiencia es distinta en cada eslabón. Así en el eslabón 
productivo es claramente negativa, al ganadero le cuesta mucho dinero producir una lana de calidad, necesita buenos alimentos, adecuado manejo del ganado, higiene de las instalaciones y la esquila.

Todo esto no es cubierto por lo que reciben por la venta de la lana, ni siquiera se cubren los gastos de la esquila. Además, dependiendo de la raza de oveja, hay lana que no es útil en la industria, y los ganaderos lo saben, y no intentan venderla, tienen que deshacerse de ella.

En el caso de la industria transformadora, el caso es similar, desde luego le aportan valor añadido al producto pero el porcentaje de material desechado es bastante alto.

En la lavadora se pierde, dependiendo del manejo del ganado en la explotación hasta un $15 \%$ si el manejo ha sido inadecuado (falta de higiene, uso de pinturas no lavables para marcar a los animales,...), en la clasificación de los vellones en función de su calidad y tipo industrial un $7 \%$ y dependiendo del tipo de lana y de su rendimiento al lavado se pierde alrededor de un $42 \%$ en el proceso de lavado.

En las fábricas cardadoras se pierde alrededor de un $10 \%$. Todas estas pérdidas hacen que para sacar beneficio al producto $\mathrm{y}$ abordar los gastos de las fábricas haya que aumentar el precio por kilogramo de lana transformada.

Aun así este precio no puede ser muy alto para ser competitivo con otras fibras y venderlo a la industria textil de los que se supone que su eficiencia económica si es positiva. La transparencia en el flujo de información, la innovación o la capacidad de adaptación son otros criterios, que en el caso del sector lanero son reducidos y se necesita del apoyo de la Administración Pública y de la colaboración de todos los miembros de la cadena para mejorar en definitiva el funcionamiento de la cadena de valor.

\section{ANÁLISIS DE LOS RESULTADOS}

La parte empírica e interesante del presente documento es la información obtenida directamente de los agentes implicados en la cadena de valor de la lana de oveja, especialmente de los productores, eslabón más afectado y transformadores.

\section{V.1 ENCUESTAS A GANADEROS}

De las encuestas realizadas a ganaderos en Madrid, Salamanca, Burgos, Zamora, Segovia y Valladolid se obtiene información relevante para el presente estudio. La mayoría son empresarios individuales con explotaciones de entre 101 y 500 cabezas de ganado.

La raza ovina más explotada por los encuestados es Assaf, cuya aptitud principal es leche y carne, le siguen Churra, Castellana y cruces, también orientadas a leche y carne. Por lo que la opinión e información de los encuestados es interesante para saber qué pasa con la lana que no tiene suficiente calidad cómo para vender a buen precio a la industria textil. El 100\% de los encuestados utiliza esquila mecánica y declaran que los costes de la esquila están entre 1 y 4 euros por oveja (alrededor de 1,50 euros).

El valor más frecuente, en cuanto a producción media de lana en la explotación se refiere, es de 1000 kilogramos y la media del peso del vellón es de 2,23 kilogramos. Cabe destacar que a pesar de que la lana no sea de alta calidad, el $100 \%$ de los encuestados vende la lana, la mayoría a cooperativas y otros a particulares o almacenistas. Aunque no seguro, la mayoría cree que el destino final de la lana que producen en sus explotaciones es textil.
El precio máximo por el que venden la lana en 1,15 euros el kilogramo, el valor más frecuente es 0,5 euros el kilogramo y el mínimo es 0,3. La totalidad de los encuestados aseguran que no cubren los gastos de la esquila con la venta de la lana. El $67 \%$ de los ganaderos preguntados afirman no recibir ningún tipo de ayuda por parte de la Administración, de hecho comentan la finalización de una subvención en Castilla y León que cubría el $50 \%$ de los gastos de la esquila, comenzó en el 2008 y terminó en 2012.

La mayoría demanda un precio más alto por su producto e incluso la búsqueda de nuevas alternativas para la lana de oveja. Sólo un $8 \%$ de los encuestados aseguró haber considerado la producción de lana ecológica, el $62 \%$ no lo ha considerado.

Las encuestas se realizaron entre finales de 2013 y principios de 2014. El rango de edad dónde se localizaba la mayoría de los encuestados era entre los 41 y los 50 años, seguidos de 51-60. El $70 \%$ de los que contestaron a la encuesta eran los propietarios de la explotación, el $91 \%$ tenía estudios primarios y el valor más frecuente de años trabajando para la explotación era 40 , de lo que se puede deducir que llevan toda la vida dedicada a la explotación de ovino.

Las respuestas proporcionadas sobre el precio percibido por la venta de la lana o el coste de la esquila, confirmaron la idea de depreciación del producto. Además algunas preguntas cualitativas acerca del destino final de su lana confirmaron la falta de transparencia en el sector.

Todos los encuestados demandan precios para la lana que al menos cubran los gastos de la esquila, o en su defecto, subvenciones por parte de la Administración. Encuestas a invernaderos.

De las encuestas realizadas a invernaderos se obtuvo información desde distintos puntos de vista, ya que la producción tenía distintos objetivos, había producción industrial con 220.000 $\mathrm{m} 2$, para investigación y para autoconsumo. Los productos más producidos eran pimiento y tomate y el $50 \%$ de los encuestados utiliza producción ecológica. Ningún encuestado ha utilizado lana de oveja como sustrato pero el $100 \%$ desearía probarlo. Fibra de coco, lana de roca, perlita, turba+arena y enarenado son los sustratos utilizados por los encuestados y el $75 \%$ estarían dispuestos a cambiar su sustrato por otro renovable y natural.

De los factores decisivos para elegir un sustrato, el precio es importante para todos los encuestados, el 75\% considera importante que aumente la producción y las que las propiedades del sustrato (absorción de agua y nutrientes o enraizamiento) sean adecuadas, el $50 \%$ considera importante que sea ecológico. Todas las encuestas se realizaron en el 2014, en Murcia, Almería, Burgos y Madrid y la mayoría de los encuestados tiene entre 41 y 50 años.

La parte empírica e interesante del presente documento es la información obtenida directamente de los agentes implicados en la cadena de valor de la lana de oveja, especialmente de los productores, eslabón más afectado y transformadores. Encuestas a ganaderos De las encuestas realizadas a ganaderos en Madrid, Salamanca, Burgos, Zamora, Segovia y Valladolid se obtiene información relevante para el presente estudio.

La mayoría son empresarios individuales con explotaciones de entre 101 y 500 cabezas de ganado. La raza ovina más explotada por los encuestados es Assaf, cuya aptitud principal es leche y carne, le siguen Churra, Castellana y cruces, también orientadas a leche y carne. Por lo que la opinión e información de los encuestados es interesante para saber qué pasa con la lana que no tiene suficiente calidad cómo para vender a buen precio a la industria textil. El $100 \%$ de los encuestados utiliza esquila mecánica y declaran que los costes de la esquila 
Bilbao, Escribano and Köhler, ITEGAM-JETIA. Vol. 04, № 13, pp 109-116. March, 2018.

están entre 1 y 4 euros por oveja (alrededor de 1,50 euros). El valor más frecuente, en cuanto a producción media de lana en la explotación se refiere, es de 1000 kilogramos y la media del peso del vellón es de 2,23 kilogramos.

Cabe destacar que a pesar de que la lana no sea de alta calidad, el $100 \%$ de los encuestados vende la lana, la mayoría a cooperativas y otros a particulares o almacenistas. Aunque no seguro, la mayoría cree que el destino final de la lana que producen en sus explotaciones es textil. El precio máximo por el que venden la lana en 1,15 euros el kilogramo, el valor más frecuente es 0,5 euros el kilogramo y el mínimo es 0,3.

La totalidad de los encuestados aseguran que no cubren los gastos de la esquila con la venta de la lana. El $67 \%$ de los ganaderos preguntados afirman no recibir ningún tipo de ayuda por parte de la Administración, de hecho comentan la finalización de una subvención en Castilla y León que cubría el $50 \%$ de los gastos de la esquila, comenzó en el 2008 y terminó en 2012.

La mayoría demanda un precio más alto por su producto e incluso la búsqueda de nuevas alternativas para la lana de oveja. Sólo un $8 \%$ de los encuestados aseguró haber considerado la producción de lana ecológica, el $62 \%$ no lo ha considerado. Las encuestas se realizaron entre finales de 2013 y principios de 2014. El rango de edad dónde se localizaba la mayoría de los encuestados era entre los 41 y los 50 años, seguidos de 51-60. El $70 \%$ de los que contestaron a la encuesta eran los propietarios de la explotación, el $91 \%$ tenía estudios primarios y el valor más frecuente de años trabajando para la explotación era 40, de lo que se puede deducir que llevan toda la vida dedicada a la explotación de ovino. Las respuestas proporcionadas sobre el precio percibido por la venta de la lana o el coste de la esquila, confirmaron la idea de depreciación del producto. Además algunas preguntas cualitativas acerca del destino final de su lana confirmaron la falta de transparencia en el sector. Todos los encuestados demandan precios para la lana que al menos cubran los gastos de la esquila, o en su defecto, subvenciones por parte de la Administración.

\section{V.2 ENCUESTAS A INVERNADEROS}

De las encuestas realizadas a invernaderos se obtuvo información desde distintos puntos de vista, ya que la producción tenía distintos objetivos, había producción industrial con 220.000 $\mathrm{m} 2$, para investigación y para autoconsumo. Los productos más producidos eran pimiento y tomate y el $50 \%$ de los encuestados utiliza producción ecológica. Ningún encuestado ha utilizado lana de oveja como sustrato pero el $100 \%$ desearía probarlo. Fibra de coco, lana de roca, perlita, turba+arena y enarenado son los sustratos utilizados por los encuestados y el $75 \%$ estarían dispuestos a cambiar su sustrato por otro renovable y natural.

De los factores decisivos para elegir un sustrato, el precio es importante para todos los encuestados, el $75 \%$ considera importante que aumente la producción y las que las propiedades del sustrato (absorción de agua y nutrientes o enraizamiento) sean adecuadas, el 50\% considera importante que sea ecológico. Todas las encuestas se realizaron en el 2014, en Murcia, Almería, Burgos y Madrid y la mayoría de los encuestados tiene entre 41 y 50 años.

Se deduce que el sector de los invernaderos es dinámico, con capacidad de adaptación y además busca sustratos naturales, renovables, que no produzcan alergias, con precio razonable, que mejore la producción y sus características organolépticas.

\section{CONCLUSIONES FINALES Y PROPUESTAS}

Como se puede deducir del estudio de la Cadena de Valor, de las opiniones y comentarios de ganaderos y del propio Ministerio de Agricultura, Alimentación y Medio Ambiente (MAGRAMA) es necesario aportar mayor valor a la lana de oveja española. Hay mucho trabajo por delante, mejoras en muchos eslabones, mayor comunicación y transparencia en la cadena, etc. La adaptación a un consumidor más exigente pasa por realizar encuestas a consumidores, desarrollo de nuevas ideas, estudios o proyectos innovadores para utilización de la lana en otros sectores distintos al textil, transmitir la información a todos los eslabones, colaboración de las Administraciones para promover el desarrollo económico en el sector, motivar a los ganaderos, etc.

\section{VI.1 NUEVAS POSIBILIDADES PARA LA UTILIZACIÓN DE LA LANA}

Hay lanas españolas sin interés para la industria textil. La investigación y el desarrollo tecnológico son necesarios para ampliar el horizonte de uso de la lana de oveja en nuestro país. Algunas aplicaciones de lana novedosas son: como aislante térmico y acústico, absorbente, para embalaje, en sectores de limpieza o para paneles de filtración. Los nuevos tratamientos con plasma mejoran algunas propiedades de las lanas tratadas y los nuevos productos textiles resultan muy atractivos para el mercado mundial. Se está produciendo un gran desarrollo de los textiles técnicos destinados a los siguientes campos: ingeniería civil (Geotextiles), vestimenta de protección personal, automoción y transporte, agricultura, jardinería, pesca, edificación, medicina e higiene, interiorismo y decoración... En todos ellos la lana puede tener aplicaciones. Hay que investigar para encontrar un futuro para las lanas que no pueden comercializarse [21].

Con este trabajo se quiere proponer la utilización de este valioso "residuo" como sustrato en cultivos forzados. Por sus características: fibrosa, ignifuga, aislante, renovable, sostenible, no produce alergias, absorbe $\mathrm{CO}_{2}$, alto contenido en Nitrógeno y otros nutrientes, capacidad de absorción de agua,... se le considera un buen candidato. Además se lleva años utilizando lana de roca (sintética) ¿por qué sintética si la podemos tener natural? Son muy conocidas en el mundo rural sus propiedades como abono, por ejemplo hace años se utilizaba la lana de oveja que salía de la peinadora de Béjar (Salamanca) para abonar los campos de cerezos del Valle del Jerte.

\section{VI.2 ¿SON LOS “WOOLVATION BLOCKS” UN FUTURO POSIBLE PARA LA LANA ESPAÑOLA?}

Gracias a la investigación y colaboración entre universidades se quiere probar la lana como soporte de cultivo aprovechando sus propiedades y características. Ya se están llevando a cabo experimentos en la Universidad Humboldt de Berlín y en los campos de prácticas de la Universidad Politécnica de Madrid. Resultados positivos ampliarían el horizonte de uso convencional para las lanas de nuestro país. Los "woolvation blocks" son una idea del Instituto de Proyectos de Ecología Agraria y Urbana (IASP), pretende ser una imitación del tipo de contenedor que se utiliza para cultivar con lana de roca, pero en vez de usar lana de roca se utilizaría lana de oveja, por lo que se debe adaptar a sus características. Sería una forma de que los ganaderos pudiesen cubrir los gastos de la esquila, recibiendo más dinero por su lana, se podría utilizar el residuo que queda tras sufrir su transformación para la industria textil, los invernaderos 
Bilbao, Escribano and Köhler, ITEGAM-JETIA. Vol. 04, № 13, pp 109-116. March, 2018.

tendrían un sustrato natural, renovable, autóctono, que no produce alergias y fácil de eliminar.

\section{RECONOCIMIENTOS}

Los autores desean agradecer al Instituto de Proyectos de Ecología Agraria y Urbana (IASP) afiliado a la Universidad Humboldt de Berlín la oportunidad brindada para trabajar sobre este tema, además de su estrecha colaboración con la Universidad Politécnica de Madrid que presta sus recursos e instalaciones para la realización del presente estudio. También a la Asociación Agraria de Jóvenes Agricultores de Madrid y Burgos por la información y colaboración prestada y a todos los que contestaron las encuestas y nos recibieron con tanta amabilidad.

\section{REFERENCIAS}

[1] “LANA. Cambie a un ambiente más sano y seguro". IWTO Federación Lanera Internacional. www.iwto.org.

[2] DE BURGOS, F.J. "Programa de Mejora de la Calidad de la Lana". Proyecto Piloto de la Red Rural Nacional "Nuevas Fórmulas de Comercialización de Subproductos Ganaderos". FEADER, ASAJA, Ministerio de Agricultura, Alimentación y Medio Ambiente, Gobierno de España 2012. Depósito legal:

M-1157-2013. Pág. 25.

[3] DE BURGOS, F.J.; RINCÓN, P. "Situación del sector, mercado y comercialización de la lana". Proyecto Piloto de la Red Rural Nacional "Nuevas Fórmulas de Comercialización de Subproductos Ganaderos". FEADER, ASAJA, Ministerio de Agricultura, Alimentación y Medio Ambiente, Gobierno de España 2010. pp 1415 .

[4] Cadena de Valor [en línea]. [ref. de 18 de agosto 2014] Disponible en: http://es.wikipedia.org/wiki/Cadena_de_valor.

[5] La Cadena de Valor de Michael Porter. Publicado por web y empresas, en enero 30, 2012 Planificación Estratégica [en línea]. [ref. de 18 de agosto 2014]. Disponible en: http://www.webyempresas.com/lacadena-de-valor-de-michael-porter/ [6] BRIZ, J.; DE FELIPE, I. Capítulo III“ Hacia una metodología GLOCAL en el estudio de la cadena de valor alimentaria" del libro: "Metodología y funcionamiento de la cadena de valor alimentaria: un enfoque pluridisciplinar e internacional. Editorial Agrícola. ISBN: 978-84-92928-23-1. pp 79-101.

[7] Censo de ovino a nivel mundial [en línea]. [ref. de 13 de febrero 2014]. Disponible en: http://faostat.fao.org/site/573/DesktopDefault.aspx?PageID=573\#anc or

[8] Censo de ovino europeo [en línea]. [ref. de 13 de febrero 2014]. Disponible http://faostat.fao.org/site/573/DesktopDefault.aspx?PageID=573\#anc or

[9] Caracterización del sector ovino y caprino en España 2012. Subdirección General de Recursos Ganaderos, Ministerio de Agricultura, Alimentación y Medio Ambiente, Gobierno de España. Disponible

http://www.magrama.gob.es/es/ganaderia/temas/produccion-ymercados-ganaderos/sectoresganaderos/ovinocaprino/default.aspx

[10] Evolución del censo de ovino en España (1990-2012) [en línea]. [ref. de13 de febrero de 2014]. Disponible en:
http://faostat.fao.org/site/573/DesktopDefault.aspx?PageID=573\#anc or

[11] DE BURGOS, F.J.; RINCÓN, P. "Situación del sector, mercado y comercialización de la lana". Proyecto Piloto de la Red Rural Nacional "Nuevas Fórmulas de Comercialización de Subproductos Ganaderos". FEADER, ASAJA, Ministerio de Agricultura, Alimentación y Medio Ambiente, Gobierno de España 2010, Pág. 30.

[12] Producción de lana grasa a nivel mundial [en línea]. [ref. de 13 de febrero de 2014]. Disponible en: http://faostat.fao.org/site/569/DesktopDefault.aspx?PageID=569anco $r$

[13] Producción lana grasa y evolución por continentes [en línea]. [ref. de 13 de febrero de 2014]. Disponible en: http://faostat.fao.org/site/569/DesktopDefault.aspx?PageID=569\#anc or

[14] Producción de lana grasa a nivel mundial por países [en línea]. [ref. de 13 de febrero de 2014]. Disponible en: http://faostat.fao.org/site/569/DesktopDefault.aspx?PageID=569\#anc or

[15] Producción europea de lana grasa por países [en línea]. [ref. de 13 de febrero de 2014]. Disponible en: http://faostat.fao.org/site/569/DesktopDefault.aspx?PageID=569\#anc or

[16] Evolución de la producción de lana grasa en Europa (1990 2012) [en línea]. [ref. de 13 de febrero de 2014]. Disponible en: http://faostat.fao.org/site/569/DesktopDefault.aspx?PageID=569\#anc or

[17] DE BURGOS, F.J.; RINCÓN, P. "Situación del sector, mercado y comercialización de la lana". Proyecto Piloto de la Red Rural Nacional "Nuevas Fórmulas de Comercialización de Subproductos Ganaderos". FEADER, ASAJA, Ministerio de Agricultura, Alimentación y Medio Ambiente, Gobierno de España 2010, pp 38-40.

[18] DE BURGOS, F.J.; RINCÓN, P. "Situación del sector, mercado y comercialización de la lana". Proyecto Piloto de la Red Rural Nacional "Nuevas Fórmulas de Comercialización de Subproductos Ganaderos". FEADER, ASAJA, Ministerio de Agricultura, Alimentación y Medio Ambiente, Gobierno de España 2010, Pág. 55.

[19] DE BURGOS, F.J.; RINCÓN, P. "Situación del sector, mercado y comercialización de la lana". Proyecto Piloto de la Red Rural Nacional "Nuevas Fórmulas de Comercialización de Subproductos Ganaderos". FEADER, ASAJA, Ministerio de Agricultura, Alimentación y Medio Ambiente, Gobierno de España 2010, pp 43-52.

[20] LANA. “Cambie a un ambiente más sano y seguro” . IWTO - Federación Lanera Internacional. www.iwto.org.

[21] MARSAL, F.; MORRAL, E.; PALET, D. "Puesta en valor de lanas y pieles de producción nacional” Trabajo solicitado por el Ministerio de Agricultura, Alimentación y Medio Ambiente, Gobierno de España. 